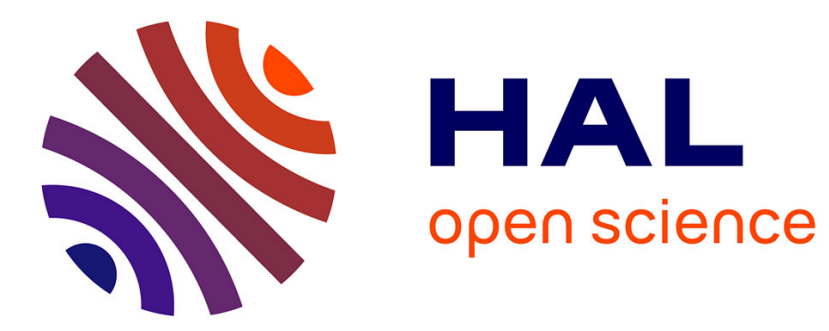

\title{
Optimized properties of chaos from a laser diode
}

Guillaume Bouchez, Tushar Malica, Delphine Wolfersberger, Marc Sciamanna

\section{To cite this version:}

Guillaume Bouchez, Tushar Malica, Delphine Wolfersberger, Marc Sciamanna. Optimized properties of chaos from a laser diode. Physical Review E , 2021, 103 (4), 10.1103/PhysRevE.103.042207. hal-03196642

\section{HAL Id: hal-03196642 \\ https://hal.science/hal-03196642}

Submitted on 13 Apr 2021

HAL is a multi-disciplinary open access archive for the deposit and dissemination of scientific research documents, whether they are published or not. The documents may come from teaching and research institutions in France or abroad, or from public or private research centers.
L'archive ouverte pluridisciplinaire HAL, est destinée au dépôt et à la diffusion de documents scientifiques de niveau recherche, publiés ou non, émanant des établissements d'enseignement et de recherche français ou étrangers, des laboratoires publics ou privés. 


\title{
Optimized properties of chaos from a laser diode
}

\author{
Guillaume Bouchez,* Tushar Malica, Delphine Wolfersberger, and Marc Sciamanna \\ Chaire Photonique, LMOPS, CentraleSupélec and Université de Lorraine, 2 Rue Edouard Belin, 57070 Metz, France
}

(Dated: March 2, 2021)

\begin{abstract}
We perform an experimental parametric study of the chaos generated by a laser diode subjected to phase-conjugate feedback. In addition to the typical figure of merit, i.e. chaos bandwidth, the corresponding spectral flatness and permutation entropy at delay is analysed. Our experimental observations reveal that the chaos can be generated with a bandwidth of $\approx 29 \mathrm{GHz}$, a spectral flatness up to 0.75 and a permutation entropy at delay up to 0.99 . These optimized performances are maintained over a large range of parameters and have not been achieved in the conventional optical feedback configuration. Interestingly, reducing the pump current reduces the chaos bandwidth while keeping the spectral flatness and the permutation entropy at delay same as observed for increased pump current. Our experimental findings are consistent with the presented numerical simulations produced using the Lang-Kobayashi model.
\end{abstract}

\section{INTRODUCTION}

Keeping a laser diode stable is usually considered as a priority. However, chaotic laser diodes can be employed in various fields, such as, encrypted communications, random number generation, and remote sensing [1-3]. The aforementioned stability of the semiconductor lasers has been perturbed to introduce chaos in the system through various popular techniques such as optical or optoelectronic feedback, optical injection, and current modulation [4]. One particular approach is the phaseconjugate feedback $(\mathrm{PCF})$ in which the light reflected into the laser is the phase-conjugate of the laser output. This configuration has shown to exhibit a variety of rich dynamics in addition to chaos, in particular, restabilization under strong feedback strength [5-7] and external cavity modes (ECMs). The latter is characterised by the self-pulsating dynamics regularly oscillating close to a multiple of the frequency of the external cavity $[7,8]$. Previously, we have also experimentally demonstrated that the PCF-generated chaos significantly enhances the chaos bandwidth when compared to the case of conventional optical feedback (COF) [9] and can exhibit highly spatiotemporally-complex outputs [10].

To quantify the chaos in the acquired time series, one widely used indicator is the chaos bandwidth. It is defined as the span of frequencies than contains $80 \%$ of the laser output power spectrum total energy [11]. Broadband chaos improves the performances of the chaotic random number generation [12] and chaos cryptography [13]. In COF, the intrinsic limit of the chaos bandwidth is given by the relaxation oscillations ( $\mathrm{RO}$ ) frequency. The chaos bandwidth can be further enhanced by dualfeedback [14] successive injections into three different laser diodes [12], mutual coupling [15] or self-interferences in a fibered Mach-Zehnder interferometer [16]. In Ref. [15], the chaos bandwidth was shown to reach up to $38.6 \mathrm{GHz}$.

\footnotetext{
* guillaume.bouchez@centralesupelec.fr
}

Further analysis of the chaotic complexity can be performed using the spectral flatness, which quantifies the distribution of the frequencies [17]. Last, the permutation entropy quantifies the entropy and measures the predictability at operating condition under consideration [18]. Previous studies on the PCF systems were limited to the evolution of chaos bandwidth $[9,19]$ or the permutation entropy [10] versus the feedback strength.

In this article, we extend prior analysis with an extensive parametric study of the chaos properties, i.e. the chaos bandwidth, the spectral flatness and the permutation entropy, as a function of the feedback strength, of the time-delay in the external cavity, and the laser pumping current. As a result, we experimentally and numerically demonstrate that the PCF approach is a robust method to generate a comprehensive chaotic system output which is broadband, flat and spatio-temporally complex. We show that the higher feedback strength improves the bandwidth of the chaos. Increasing the pump current increases the chaos bandwidth while the spectral flatness and the permutation entropy remain consistently high, independent of the other parametric changes. However, the time delay has negligible impact on the PCF-induced chaos. We, therefore, demonstrate that by tuning the experimental parameters the user can obtain highly complex chaos and simultaneously adjust its chaos bandwidth.

We observe an optimal bandwidth of $28.7 \mathrm{GHz}$, an entropy at delay up to 0.99 and a flatness up to 0.75 , and these high values are maintained over a large range of parameters. Our experiment shows, best to our knowledge, the best chaos performance in the simplest configuration of a laser diode with a single optical feedback, i.e., without any additional coupling or injection.

\section{EXPERIMENTAL FINDINGS}

\section{A. Principle of the experiment}

Our experimental setup is presented in Fig. 1 and is similar to the one in Refs. $[7,10,19]$. We use a 


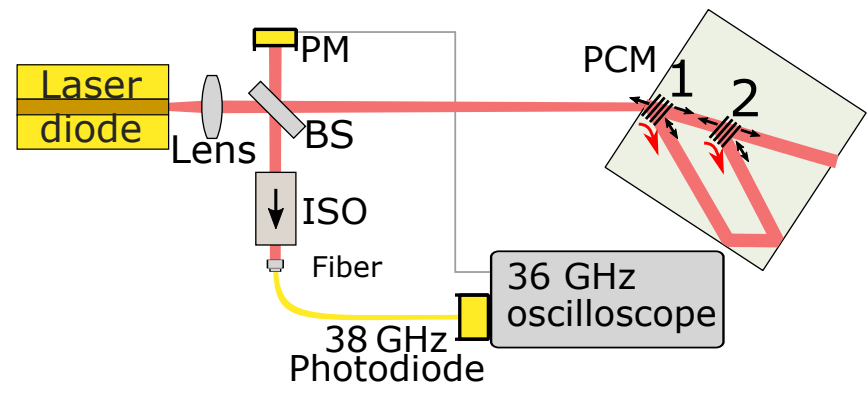

FIG. 1. Setup of the experiment.

commercial Fabry-Pérot laser diode emitting at $852 \mathrm{~nm}$, model JDS-Uniphase SDL-5420. Its threshold current is $13.9 \mathrm{~mA}$ and we operate it between 30 and $80 \mathrm{~mA}$. At $80 \mathrm{~mA}$, the laser output power is $54.4 \mathrm{~mW}$. $20 \%$ of the laser output is sent to a high bandwidth photodiode, through an optical fiber. A Faraday isolator prevents parasitic reflections from light coupling into the fiber. The laser output light is then focused by a lens into a rhodium-doped barium titanate $\left(\mathrm{BaTiO}_{3}\right)$ crystal of dimensions $5 \mathrm{~mm} \times 5 \mathrm{~mm} \times 5 \mathrm{~mm}$ that works as a phaseconjugate mirror. The phase-conjugate feedback follows the inverse path of the laser output beam and enters back into the laser internal cavity. $20 \%$ of this feedback light is sent to a power meter to know the feedback strength. It is defined as the ratio of the free running laser output to the power of the light sent back into the laser facet. We set the distance between the mirror and the laser at two different values, at 53.2 and $14.7 \mathrm{~cm}$. These values correspond to the round-trip delays of $3.55 \mathrm{~ns}$ and $0.98 \mathrm{~ns}$ and external-cavity frequencies of $282 \mathrm{MHz}$ and $1.02 \mathrm{GHz}$ respectively. These values were verified by an observation of the autocorrelation function of the time series. Due to the chosen experimental configuration, the delay length is always inferior to the period of the relaxation oscillations frequency implying our study lies in the so-called long external cavities [20].

The laser dynamics is analyzed by a high bandwidth photodiode (Newport 1474-A, AC coupled, 3dBbandwidth of $38 \mathrm{GHz}$ ) connected to an oscilloscope (Teledyne LeCroy 10-Zi-A) with a bandwidth of $36 \mathrm{GHz}$ and a sampling rate of $80 \mathrm{GSample/s}$. The output of the power meter is also connected to the oscilloscope. We record $1-\mu$ s long time series.

The phase-conjugate mirror principle is shown on the right of Fig. 1. The beam enters the crystal and part of it is deviated by beam fanning, which is a photorefractive effect taking place inside the crystal. The deviated light is reflected by the edges of the crystal. Thus, the light deviated at point 1 arrives at point 2 . Similarly, the light deviated at point 2 arrives at point 1 . At point 1, four wave-mixing occurs between the non-deviated beam, the beam deviated at point 2 , and the beam deviating at point 1 . The fourth beam is the counter-propagative phase-conjugate of the input beam. Similar wave-mixing occurs at point 2 . This configuration is named "cat" mirror $[21]$.

\section{B. Various chaotic dynamics generated by the system}

Figure 2 illustrates several dynamics of a PCF system. Figures 2 (a)-(e) show a $120 \mathrm{~ns}$ subset of the acquired time-series measured on $120 \mathrm{~ns}$ (on a total length of $1 \mu \mathrm{s}$ ). A zoom on a time-slot of 10 ns is also displayed in Figs. 2 (f)-(i).

Figures 2 (a) and (f) show the case of low feedback strength. Irregular pulsations are found with intensity peaks generally separated by a time-span of about $160 \mathrm{ps}$ to $200 \mathrm{ps}$ (see for instance the oscillations between $42 \mathrm{~ns}$ and $43 \mathrm{~ns}$ ). However, faster dynamics can also be observed on the same time traces. They are particularly visible between 43 and $44 \mathrm{~ns}$. Such dynamics match earlier observations of chaos from undamped relaxation oscillations $[6,19,22]$. Indeed, in case of an increased feedback strength, the system undergoes a first bifurcation into leading to a limit cycle at the relaxation oscillation frequency $[5,23]$.

Figures $2(\mathrm{~b})$ and $(\mathrm{g})$ are for higher feedback strengths. One can notice the time traces displays both "slow" $(\approx 200 \mathrm{ps})$ and faster variations, hence the chaos encompasses a wider range of time scales. As the feedback is further increased in Figs. 2 (c) and (h), the laser is at the edge of a dynamical change and follows an intermittent destabilized limit cycle, mixing chaos and regular oscillations. Between $18 \mathrm{~ns}$ and $41 \mathrm{~ns}$, the output power is close to periodicity, with a period of approximately $150 \mathrm{ps}(6.8 \mathrm{GHz})$. While at longer times the signal is highly chaotic. The system goes back and forth between chaos and quasi-periodicity and an analysis of the full time series confirms it. Figure $2(\mathrm{~h})$ is centered on the bifurcation and shows that the quasi-periodic signal is interrupted for a few picoseconds every nanosecond. This duration of $1 \mathrm{~ns}$ is a signature of the delay time. The system, therefore, enters a dynamical regime where both relaxation oscillations and external cavity time-scales interplay.

The time series in Fig. 2 (d) shows an external cavity mode (ECM), i.e. a limit cycle solution that is signature of the PCF $[7,8]$. The output power of the laser oscillates at a period equal to $111 \mathrm{ps}(9.02 \mathrm{GHz})$, which is about the ninth harmonic of the frequency of the external cavity $(9.18 \mathrm{GHz})$. By comparison, the period of the ROs at that current is $208 \mathrm{ps}(4.8 \mathrm{GHz})$. Such high-frequency pulsed solution is an example of the previously introduced ECMs, which are peculiar solutions of the PCF system.

Figures 2 (e) and (j) show oscillations at various timescales. These oscillations are often observed to be faster than in Fig. 2 (b). However, this time series displays a strong signature of the time delay. Short pulses are indeed sometimes generated at a repetition rate equal to $2.55 \mathrm{~ns}$ (the delay used for this specific measurement), 

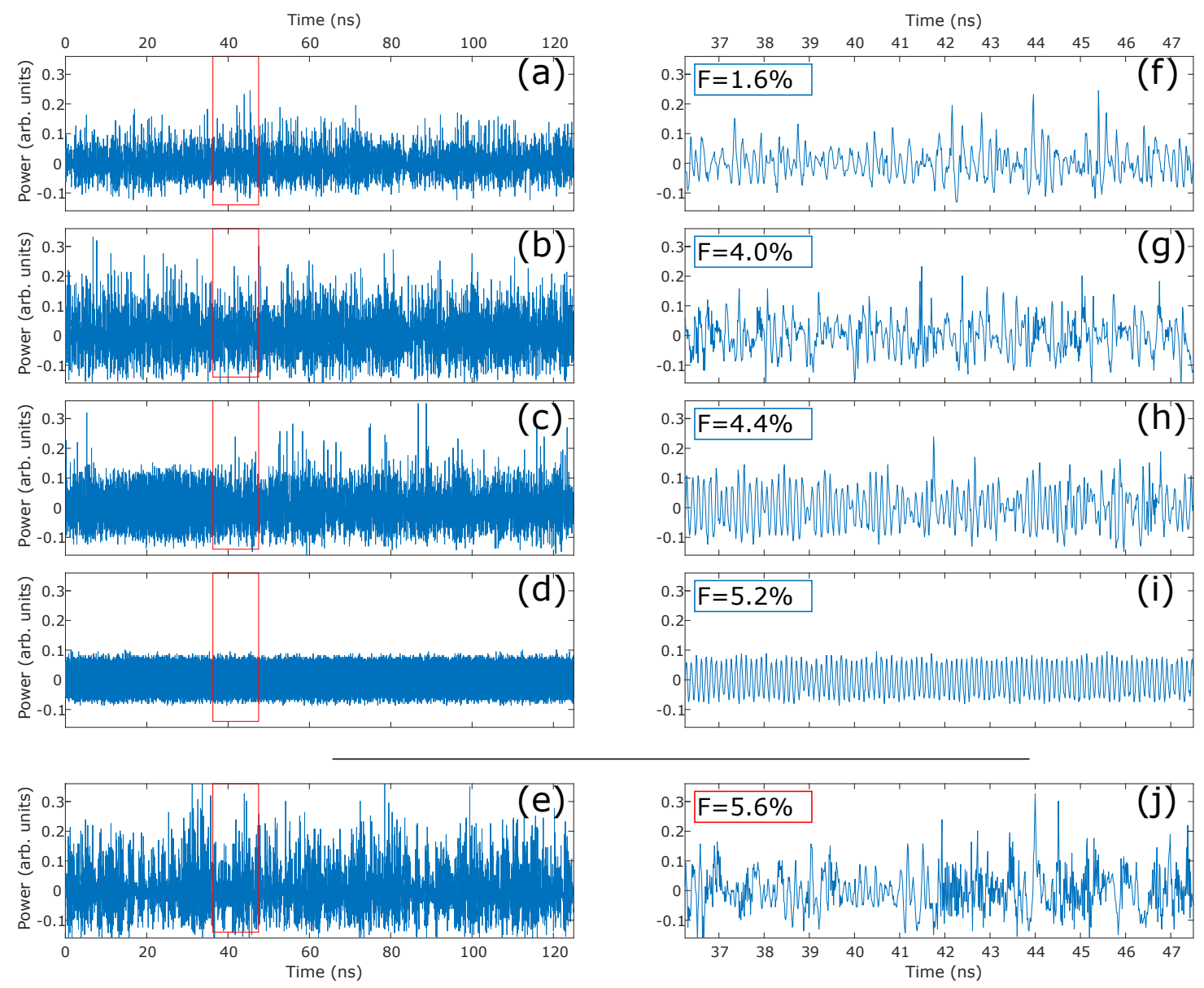

FIG. 2. Time traces of the laser output power at several values of feedback strength, delay and pump current. (a) to (d), are obtained under a pumping current of $80 \mathrm{~mA}$ and a delay length of $0.98 \mathrm{~ns}$, while (e) was measured with a pump of $50 \mathrm{~mA}$ and a delay length of 2.55 ns. (f)-(j) are zooms on the region marked with a red square on (a)-(e). Feedback strength of (e) is $5.6 \%$. The arbitrary units of (a)-(d)/(f)-(i) are similar but a different scale is used on (e) and (j).

for instance between 84 ns and 102 ns. A very limited number of time series shows such chaotic signatures and all of them simultaneously have broadband chaos, as confirmed in the following text.

\section{Quantifying chaos properties}

The large diversity of the chaos reported in Fig. 2 calls for a more quantitative analysis of the chaos properties. Therefore, several indicators have been introduced: the chaos bandwidth, the spectral flatness and the permutation entropy.

The chaos bandwidth is defined as the span of frequencies than contain $80 \%$ of the total energy of the RF spectrum[11]. With this definition, a broadband chaos will have strong preference to higher frequencies.

To verify whether the frequencies are broadly distributed, the spectral flatness $S F$ is defined as the ratio of the arithmetic mean by the geometric mean of the $80 \%$ highest energy part of the spectrum [17]:

$$
S F=\frac{\prod_{n=1}^{N} P\left(f_{n}\right)^{1 / N}}{\sum_{n=1}^{N} \frac{P\left(f_{n}\right)}{N}}
$$

where $P\left(f_{n}\right)$ is the $n$-th discrete component of the power spectral density at the frequency $f_{n}$ and where $f_{N}$ is the frequency of the chaos bandwidth $\left(\sum_{n=1}^{N} P\left(f_{n}\right)\right.$ contains $80 \%$ of the total spectrum energy). A spectral flatness of 1 is associated with a flat spectrum, for example a theoretical white noise, while the spectral flatness tends to 0 for spiky signals [24]. Another definition, which only considers the span in $\mathrm{dB}$ between the maximum and the minimum has been introduced in other works [12]. But such definition does not satisfy the definition in signal processing and does not apply to the time series analysis. The upper limit of the spectral flatness from the noise generated solely by the oscilloscope fluctuates between 0.83 and 0.84 , and, therefore, the maximum value we can measure. Indeed, in practice, the power spectral density of a white noise is distributed around a constant value with a non-zero standard deviation. Therefore, the 
maximum spectral flatness is strictly inferior to 1 .

Last, we introduce the permutation entropy (PE) as a precise indicator of the complexity of a time trace $[25,26]$. We quantify complexity by measuring the recurrence and the probability distribution of the carefully chosen much smaller subsets of the time-series referred to as ordinal patterns $(\Delta)$. These ordinal patterns are essentially similar-patterned in relative amplitudes between adjacent data points grouped together. The algorithm, as explained in [25, 26] and previously used in our work presented in [10], is calculated for two given user-defined parameters: the PE-delay $(\theta)$ and the ordinal pattern length (D). Mathematically, the normalized PE $\left(\rho_{\theta}\right)$ for a given probability distribution ' $p$ ' associated with ' $i$ ' integral number of ordinal patterns and timescale ' $\theta$ ':

$$
\rho_{\theta}=\frac{-1}{\ln (D !)} \sum_{i=1}^{D !} p\left(\Delta_{i}\right) \ln \left[p\left(\Delta_{i}\right)\right]
$$

A maximum of $D$ ! number of ordinal patterns may be constructed given the total length of the time-series is much larger than the ordinal pattern length, i.e. $N>>$ $D$. The result will be a value of $0 \leq \rho_{\tau} \leq 1$ with zero signifying complete predictability while one indicates complete stochasticity.

\section{Analysis of PCF chaos}

Figure 3 shows the power spectra of the time series we studied in Fig. 2. The drop-off of the spectra at a frequency of $38 \mathrm{GHz}$ is due to the maximum bandwidth of the oscilloscope. Chaotic spectra from lasers with feedback display resonances at multiples of the frequency associated with the external cavity, $1.02 \mathrm{GHz}$ for Figs. 3 (a)-(d) and $292 \mathrm{MHz}$ for Fig. 3 (e). These resonances will impact on the complexity of the chaos. But the spectral flatness will indicate the strength of these resonances. We use the method of Ref. [19] to measure the chaos bandwidth. We removed the part of the spectrum whose components have a power spectral density less than 3-dB superior to the noise levels. The blue part (respectively red) of the spectra contain $80 \%$ (respectively 20\%) of the remaining energy.

The ECM observed in Fig. 3 (d) oscillates at a frequency of $9.02 \mathrm{GHz}$. This frequency equals 8.84 times the frequency of the external cavity $(1.02 \mathrm{GHz})$, which is about the ninth harmonics of the frequency of the external cavity. This observation agrees with earlier theoretical predictions that tell us that the frequency of the ECMs is close but not always equal to the harmonics of the external cavity frequency, and even more, varies with the feedback strength [27, 28].

The chaos in Fig. 3 (a) is obtained at low feedback strength and its frequency spectrum is similar to the chaos obtained with COF[15]. Most of the energy is centered around the RO frequency $(4.8 \mathrm{GHz})$ and the chaos bandwidth is $13.6 \mathrm{GHz}$. This is comparable to what one
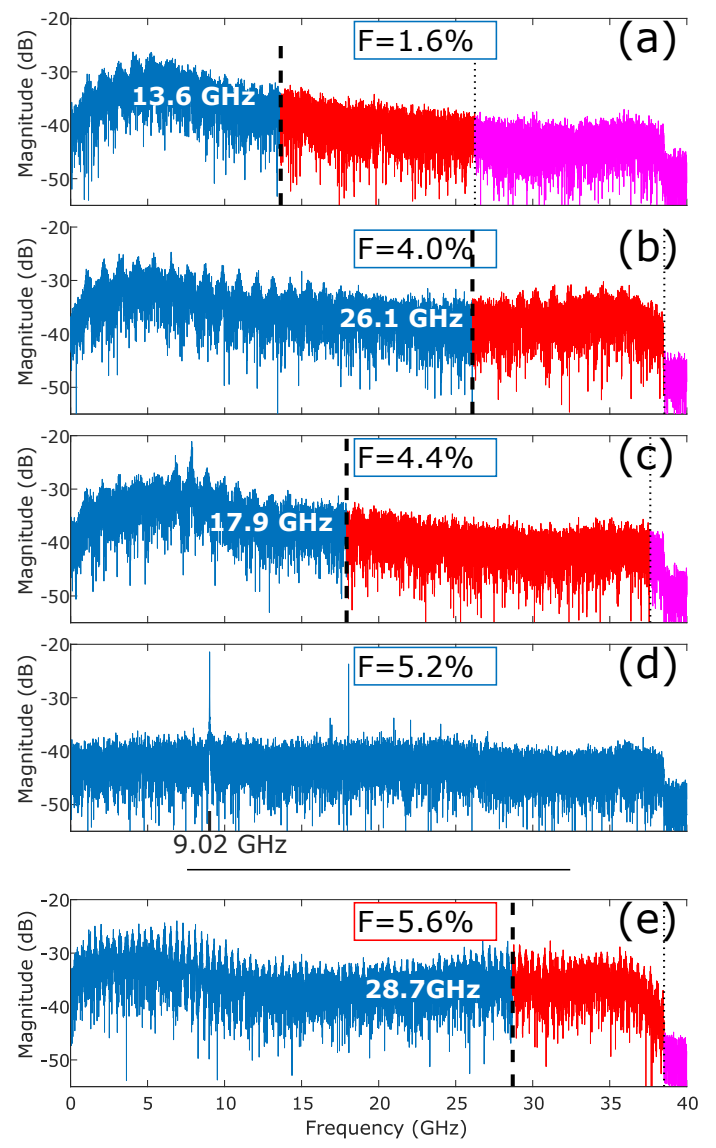

FIG. 3. Spectra of the laser output powers, corresponding to the time traces of Fig. 2, at several values of feedback strength, delay and pump current. (a) to (d), are obtained under a pumping current of $80 \mathrm{~mA}$ and a delay length of $0.98 \mathrm{~ns}$, while (e) was measured with a pump of $50 \mathrm{~mA}$ and a delay length of 2.55 ns. Feedback strength of (e) is $5.6 \%$. The blue part of spectrum contains $80 \%$ of the total energy and the chaos bandwidth is indicated in white. The magenta region is the low energy part of the spectrum and is therefore not taken in account in the calculations of chaos bandwidth.

would achieve if the PCF was replaced by a COF. However, the spectral flatness, 0.74, shows that the energy is well distributed in this limited range of frequencies. The chaos of Fig.3 (b) shows higher frequencies. The chaos bandwidth rises to $26.1 \mathrm{GHz}$ and the spectral flatness equals 0.71 . It should be noted that these values are significantly high when considering the high bandwidth, since high bandwidths are generally associated with limited spectral flatness in older works [17]. The spectrum shown in Fig.3 (c) is remarkable because it features clear peaks at $6.80 \mathrm{GHz}$ and $7.86 \mathrm{GHz}$ alongside standard chaotic spectrum. Its spectral flatness equals 0.71 .

The chaos bandwidth of Fig. 3 (e) equals to $28.7 \mathrm{GHz}$ and is even higher than in Fig. 3 (b), and equals $80 \%$ of the bandwidths of the oscilloscope $(36 \mathrm{GHz})$, hence is the maximum value we can measure. The associated spectral flatness remains high, 0.72. The spectrum also exhibits 


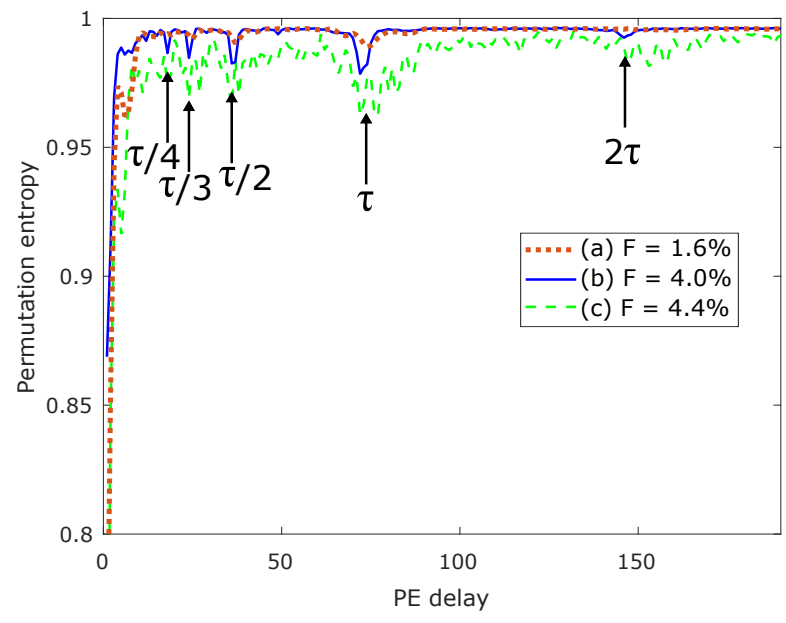

FIG. 4. Evolution of the permutation entropy as a function of PE delay for the three time traces obtained at feedback strengths of $1.6 \%$ (green dashed line), $4.0 \%$ (red dotted line) and $4.4 \%$ (green dashed line). The multiples or sub-multiples of the external cavity delay are indicated with arrows. PE delay is expressed in multiples of the sample time (12.5 ps) and $\tau$ is here the external cavity round-trip time.

a bump in the frequencies between $25 \mathrm{GHz}$ and $30 \mathrm{GHz}$ and that bump explains the exceptional value of chaos bandwidth we report. A similar bump was observed in some spectra of Ref. [10], where we reported values of chaos bandwidth up to $30 \mathrm{GHz}$.

Figure 4 shows the permutation entropies associated with the time traces of Fig. 3 (a)-(c). Values are calculated with a pattern length of $D=7,80000$-points long time series and the PE delay $(\theta)$ varies from 1 to 190 . The permutation entropy of the ECM of Fig. 3 (d), at a feedback strength of $5.2 \%$, is very low and oscillates around 0.6 when varying the PE delay. For the remaining operating conditions, signatures are observed when PE delay $\theta$ equals a submultiple of the external cavity delay: $\theta=\tau / 4, \theta=\tau / 3, \theta=\tau / 2, \theta=\tau$ and $\theta=2 \tau$. The time series obtained at a feedback strength of $4.0 \%$ (in blue), has a lower chaos bandwidth and stronger temporal signatures than the time series obtained at a feedback strength of $1.6 \%$ (in red), while the PE remains high. However, the time series at a feedback strength of $4.4 \%$ (in green) presents a lower permutation than the other cases. The mixed ECM-chaos dynamics, therefore, reduces the complexity. In the following analysis of the permutation entropy, we will only consider the PE for $\theta$ close to the external cavity delay $\tau$. Since for that $\theta$, the $\mathrm{PE}$ shows the strongest signature of the external cavity delay, $\mathrm{PE}$ at $\theta \approx \tau$ will be referred simply as $\operatorname{PE}(\tau)$ here onwards.
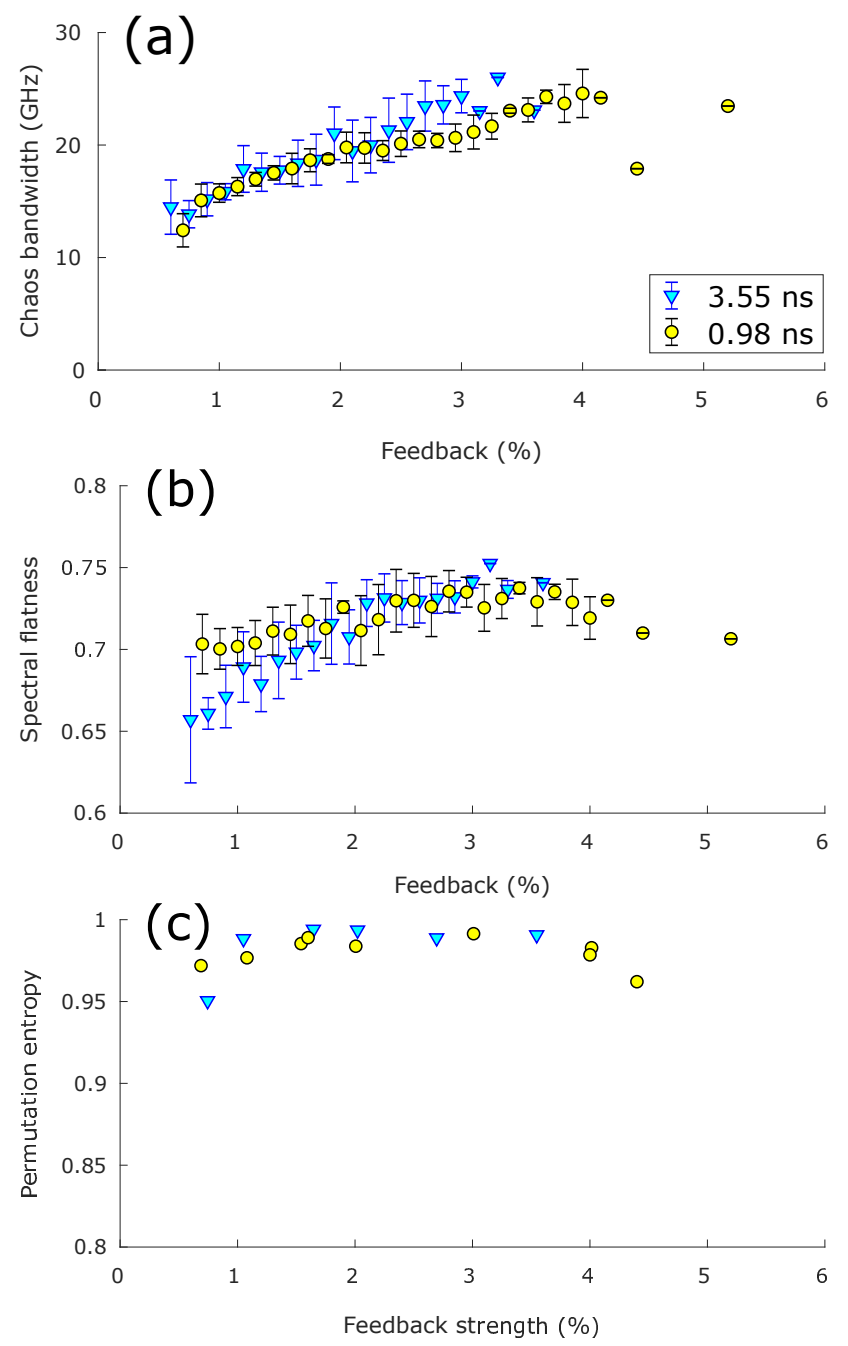

FIG. 5. Evolution of (a) the average chaos bandwidth, (b) the average spectral flatness and (c) the $\mathrm{PE}(\tau)$ versus the feedback strength. The pump current is set at $80 \mathrm{~mA}$. Experiments have been performed with two values of delay: $3.55 \mathrm{~ns}$ (blue triangles) and $0.98 \mathrm{~ns}$ (yellow circles).

\section{E. Influence of the feedback strength and of the delay length}

We show in Fig. 5 (a) the experimental trend of the chaos bandwidth versus the feedback strength. Furthermore, Figs. 5 (b)-(c) show the evolution of the spectral flatness and of the $\operatorname{PE}(\tau)$ with the feedback strength and for both the external cavity delays. The pump current is fixed at $80 \mathrm{~mA}$. The natural slow variations of the reflectivity of the mirror due to nonlinear optics taking place inside the crystal allow us to address various values of feedback strength $[7,19]$. The range of the feedback strength variations can be tuned with the geometry of the crystal [29] and we have observed that in our experiment. The chaos properties are shown for the feedback strength values being separated by $0.15 \%$. Each point is accompanied with a bar indicating the standard de- 
viation when computing the property for the different operating conditions. The $\operatorname{PE}(\tau)$ being longer to calculate, we selected a smaller number of time series and calculated their $\operatorname{PE}(\tau)$. The multi-stability between the different dynamics is indicated by error bars as it shows the variations in the chaos properties in the PCF system $[23,30]$. However, the standard deviation of the chaos bandwidth on each range of feedback is rather limited. The variations for the spectral flatness are stronger, especially for the $3.55 \mathrm{~ns}$ delay at low feedback strength. However, the overall trend indicates that the variations in the spectral flatness decreases as the feedback strength increases.

Both, the chaos bandwidth and the spectral flatness increases with the feedback strength until the feedback strength equals $3 \%$. Following that, for values of feedback strength higher than $3 \%$, the spectral flatness decreases with the feedback strength while the chaos bandwidth saturates at high values, up to $26 \mathrm{GHz} . \mathrm{PE}(\tau)$ rises with the feedback strength and reaches a plateau at a feedback strength of $1.5 \%$, then decreases slightly. The maximum value of $\operatorname{PE}(\tau)$ is 0.994 . These drops in the $\operatorname{PE}(\tau)$ and in the spectral flatness are related to the onset of the self-pulsing ECMs starting when the feedback strength is greater than $2.5 \%$.

\section{F. Influence of the pump current}

Figure 6 shows the evolution of chaos bandwidth, spectral flatness, and the $\mathrm{PE}(\tau)$ when varying the pump current from $40 \mathrm{~mA}$ to $80 \mathrm{~mA}$. We trace in Fig. 6 (a) the averaged value of the chaos bandwidth for all the time series whose feedback strengths are in the following ranges: 0.45 to $0.55 \%$ (blue diamonds), 0.95 to $1.05 \%$ (magenta diamonds), 1.45 to $1.55 \%$ (black diamonds), 2.45 to $2.55 \%$ (green diamonds) and 3 to $3.5 \%$ (red diamonds), versus the pump current. Figure 6 (b) similarly displays the averaged value of the spectral flatness on several intervals of feedback strengths. Finally, we give in Fig. 6 (c), the evolution of the $\operatorname{PE}(\tau)$ versus the pump current, for feedback strength in the ranges $0.45 \%$ to $0.55 \%$ and $2.45 \%$ to $2.55 \%$. The delay length is here fixed at $52.2 \mathrm{~cm}$ (round-trip time of $3.55 \mathrm{~ns}$ ).

We observe that the chaos bandwidth generally increases with the pump, with some exceptions, such as a remarkable value of $22 \mathrm{GHz}$ of average chaos bandwidth measured at a pump of $40 \mathrm{~mA}$ and a feedback strength $\approx 2.5 \%$. The unusual variations of the chaos bandwidth versus the pump current for a feedback strength of $2.5 \%$ can be explained by the apparition or not of the mixed chaos and ECMs dynamics described in section II B which impacts the chaos properties.

However, the evolution of the spectral flatness is more complex. For a pump current of $40 \mathrm{~mA}$, the spectral flatness decreases with an increasing feedback strength. The high spectral flatness for a feedback strength of $0.45 \%$ at $40 \mathrm{~mA}$ is probably due to the smaller variations of the
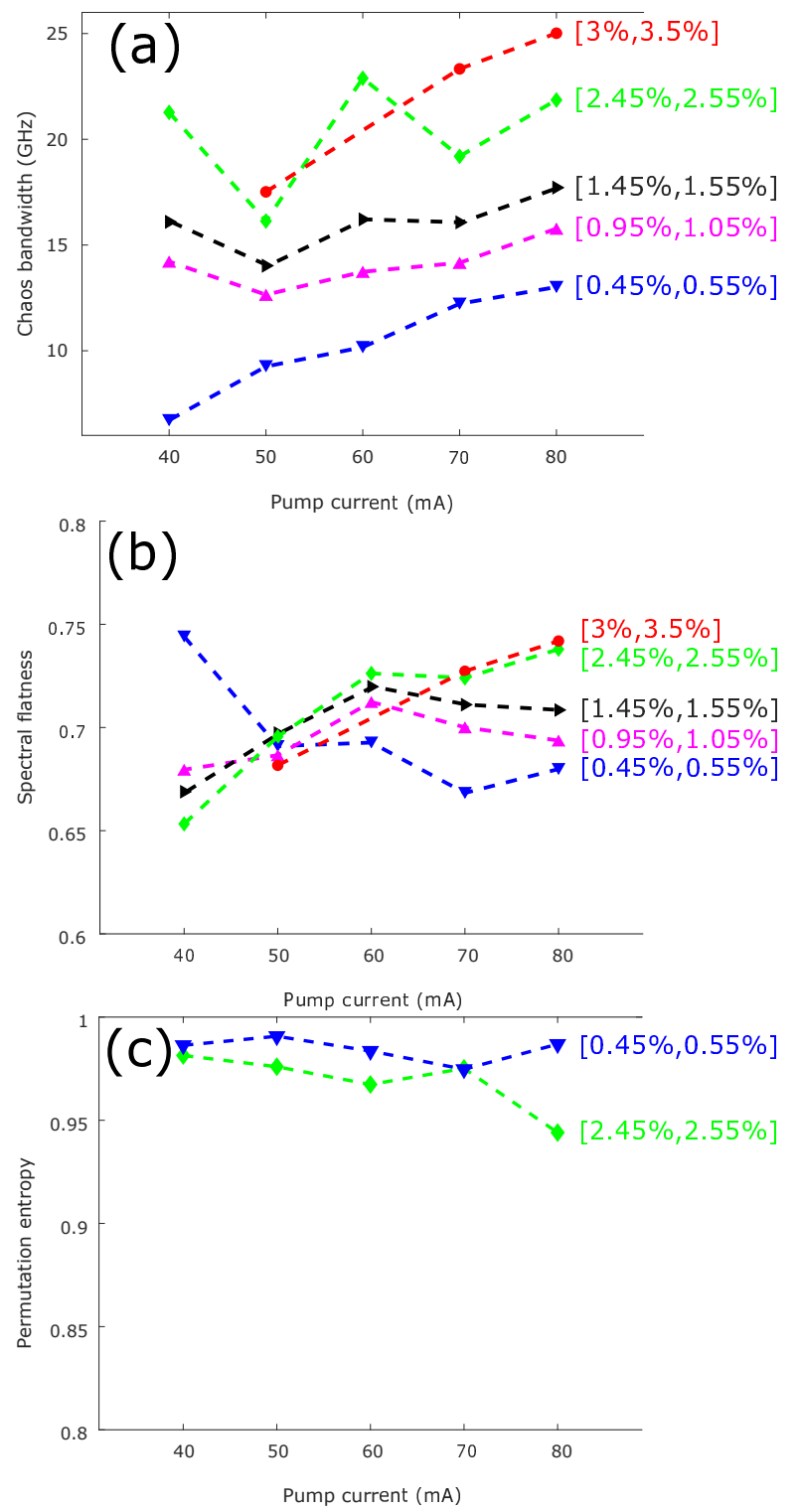

FIG. 6. Measured evolution of (a) the chaos bandwidth, (b) the spectral flatness and the (c) the $\operatorname{PE}(\tau)$ versus the pump current, for feedback strength in 5 various intervals, $[0.45,0.55 \%]$ (blue diamonds), $[0.95 \%, 1.05 \%]$ (magenta diamonds), [1.45\%, 1.55\%] (black diamonds), [2.45\%, $2.55 \%]$ (green diamonds) and [3\%,3.5\%] (red diamonds). The delay is equal to $3.55 \mathrm{~ns}$.

laser output power, since the pump current is closer to the threshold. In this case the measurement of the laser output power is close to the electronic noise of the oscilloscope.

The $\operatorname{PE}(\tau)$ always remains high for all pump currents. Slightly higher $\operatorname{PE}(\tau)$ values are observed for smaller feedback strengths and do not decrease when varying the pump current. The main finding here is that the complexity will not significantly change if the chaos bandwidth increases due to an increase in the pump current, suggesting that the chaos generated by PCF chaos is both 
complex and broadband. In COF, the chaos bandwidth is lower than the PCF chaos bandwidth for most of low values of feedback $[9,17]$. As the feedback increases, both simulations [17] and experiments [31], observe a decrease of the $\operatorname{PE}(\tau)$.

\section{NUMERICAL ANALYSIS}

\section{A. Numerical model}

To simulate laser dynamics with phase-conjugate feedback, we use a Lang-Kobayashi model, modified for phase-conjugate feedback [32]:

$$
\begin{gathered}
\dot{E}(t)=(1+i \alpha) E(t) N(t)+\gamma F(t) \\
T \dot{N}(t)=P-N(t)-(1+2 N(t))|E(t)|^{2} \\
\tau_{R} \dot{F}(t)=E^{*}(t-\tau)-F(t)
\end{gathered}
$$

$E(t)$ is the complex normalized electric field of the laser, $F(t)$ is the complex normalized feedback field and $N(t)$ is the real normalized carrier density. $t$ is the time, $\tau$ is the round-trip time in the external cavity, $T$ is the electron lifetime, all times being expressed as multiples of the photon lifetime $\tau_{p} . \gamma$ is the dimensionless feedback strength, $\alpha$ is the linewidth enhancement factor and $P$ is a pump parameter. There is no phase-shift in the PCF equations because the phase-shift of the forward propagation is canceled by the phase-conjugate backward propagation [33].

Phase-conjugate mirrors do not generate instantaneously the phase-conjugated beam. To account this in simulations, the finite depth penetration time, $\tau_{R}$, has been added [32, 34]. It filters the phase-conjugate feedback field and is directly proportional to the interaction length in the mirror. We investigated the influence of $\tau_{R}$ in Ref. [19] and demonstrated that increasing $\tau_{R}$ forces the chaos bandwidth to saturate. We now extend this analysis to additional parameters. In the experiment that we presented in section II A, we are using a $5 \mathrm{~mm}$ $\times 5 \mathrm{~mm} \times 5 \mathrm{~mm}$ cube and the associated finite depth penetration $\tau_{R}$ is $50[32]$. We set $\mathrm{T}=1200$ and $\tau_{p}=1.4 \mathrm{ps}$, as in Ref. [32]. To match our experimental observations of the relaxation oscillations frequencies, we choose:

$$
P=0.2 \frac{I-I_{t h}}{I_{t h}}
$$

$I$ being the pump current and $I_{t h}$ the threshold current (13.9 $\mathrm{mA}$ here). $\tau_{R}$ is set to 50 [7]. We choose $\alpha=3$ as typical for a laser diode.

We simulate 20000-points long time series, with an integration step equal to the photon lifetime $\tau_{p}$. We simulate the laser system for increasing values of feedback strength, each simulation being initialized with the result of the previous simulation. To avoid the system dwelling on seldom stable dynamics, we add a gaussian noise with a standard deviation of $10^{-12}$ to the intensity of the output field. In the following, we focus on the properties of the chaotic outputs.

\section{B. Influence of the feedback strength and of the delay}
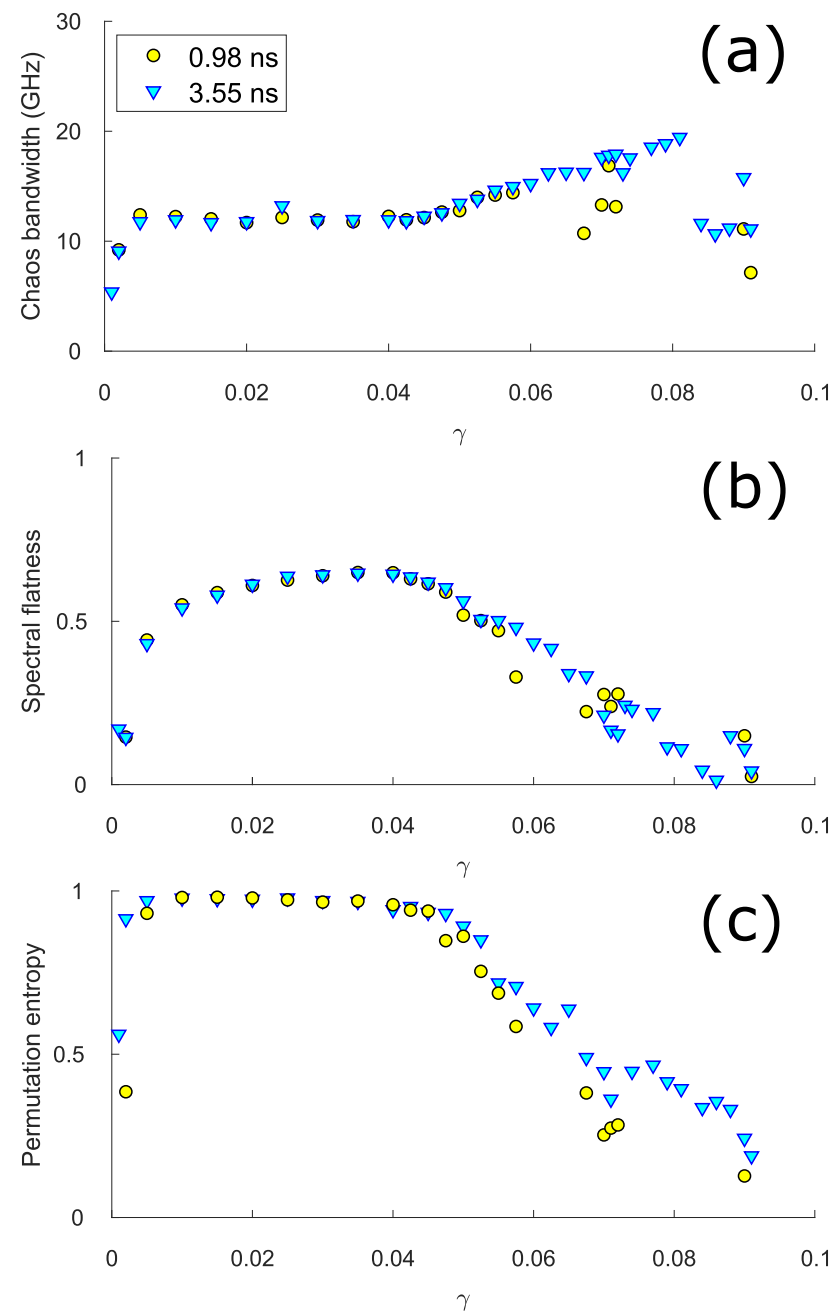

FIG. 7. Theoretical evolution of (a) the chaos bandwidth, (b) the spectral flatness and (c) the $\operatorname{PE}(\tau)$ versus the feedback strength. The pump current $I=80 \mathrm{~mA}$. Simulations have been performed with two values of delay: $3.55 \mathrm{~ns}$ (yellow circles) and 0.98 ns (blue inverted triangles).

We first compare our experimental results of Fig. 5 with Fig. 7 where the simulated chaos bandwidth (a), spectral flatness (b) and $\operatorname{PE}(\tau)$ (c) are displayed versus the feedback strength $\gamma$. To compare with the dynamical regimes found experimentally in Fig. 5, we note that the appearance of the ECMs is at $\gamma \approx 0.06$ in simulations and at $\approx 2.5 \%$ in experiment. The restabilization to the steady state numerically occurs at $\gamma \approx 0.1$ and 
experimentally at a feedback strength of approximately $5 \%$.

Simulations predict a qualitatively similar evolution of the chaos bandwidth regardless the delay, with some values of feedback creating small variations in the bandwidth, especially for $\gamma>0.06$. The evolution described in Ref. [19] is also confirmed by the argument that the chaos bandwidth of a filtered PCF system increases and then saturates when approaching the restabilization of the laser (here $\gamma=0.085$ ).

$\operatorname{PE}(\tau)$ and spectral flatness are found to be rather insensitive to the changes of the time delays, as consistent with our experimental observations. Furthermore, the $\operatorname{PE}(\tau)$ and flatness increase with $\gamma$ and reach high values. They decrease to much smaller values for large $\gamma$ compared to our observations in experiment. From $\gamma=0.04$ onwards, both $\operatorname{PE}(\tau)$ and spectral flatness fall into low values. Similar to the experiment, this drop can be related to the onset of ECMs with a stronger drop and the spectral flatness of less than 0.01 with the $\operatorname{PE}(\tau)$ of 0.2 . We explain this discrepancy by the mixed ECM+chaos dynamics found experimentally in that range of $\gamma$ and not observed in simulations. The origin of such dynamics remains unclear and may relate to the multistability of ECMs driven by noise $[23,30]$. Intermittent switching between dynamical solutions like observed experimentally help distributing the energy among different frequencies and maintaining a large number of different patterns. Hence this helps maintaining a good flatness and $\mathrm{PE}(\tau)$. Simulations in that range of $\gamma$ show instead fully chaotic states whose spectrum are centered around the frequency of the ECMs, reducing therefore flatness and $\mathrm{PE}(\tau)$.

\section{Simulated influence of the pump}

We show in Fig. 8 the simulated evolution of the chaos bandwidth versus the current $I$ for the following values of feedback strength: $\gamma=0.1, \gamma=0.06, \gamma=0.04, \gamma=0.06$, $\gamma=0.08$. To capture the main features, we averaged chaos bandwidth and spectral flatness from ten simulations with random initialization. Similarly, PE is calculated for two different simulations. The observations from the experiment of Fig. 6 are confirmed by the simulation. Thus, the chaos bandwidth increases with the pump current. In particular, the increase in the chaos bandwidth with the pump current is stronger when $\gamma$ is higher. This was also observed in Fig. 6 (i.e., in experiment) for feedback strength between $3 \%$ and $3.5 \%$. The spectral flatness is independent from the change of pump current. Similarly for lower feedback strengths, increasing the pump current even slightly, decreases the spectral flatness. This confirms our experimental findings. On the other hand, the pump current has little influence on the $\operatorname{PE}(\tau)$ at low values of $\gamma$. However, if $\gamma \geq 0.06$, an increase of the current strongly enhances the $\operatorname{PE}(\tau)$. The value of $\gamma$ associated with the onset of ECMs decreases with the pump current [28]. Hence the drop of spectral
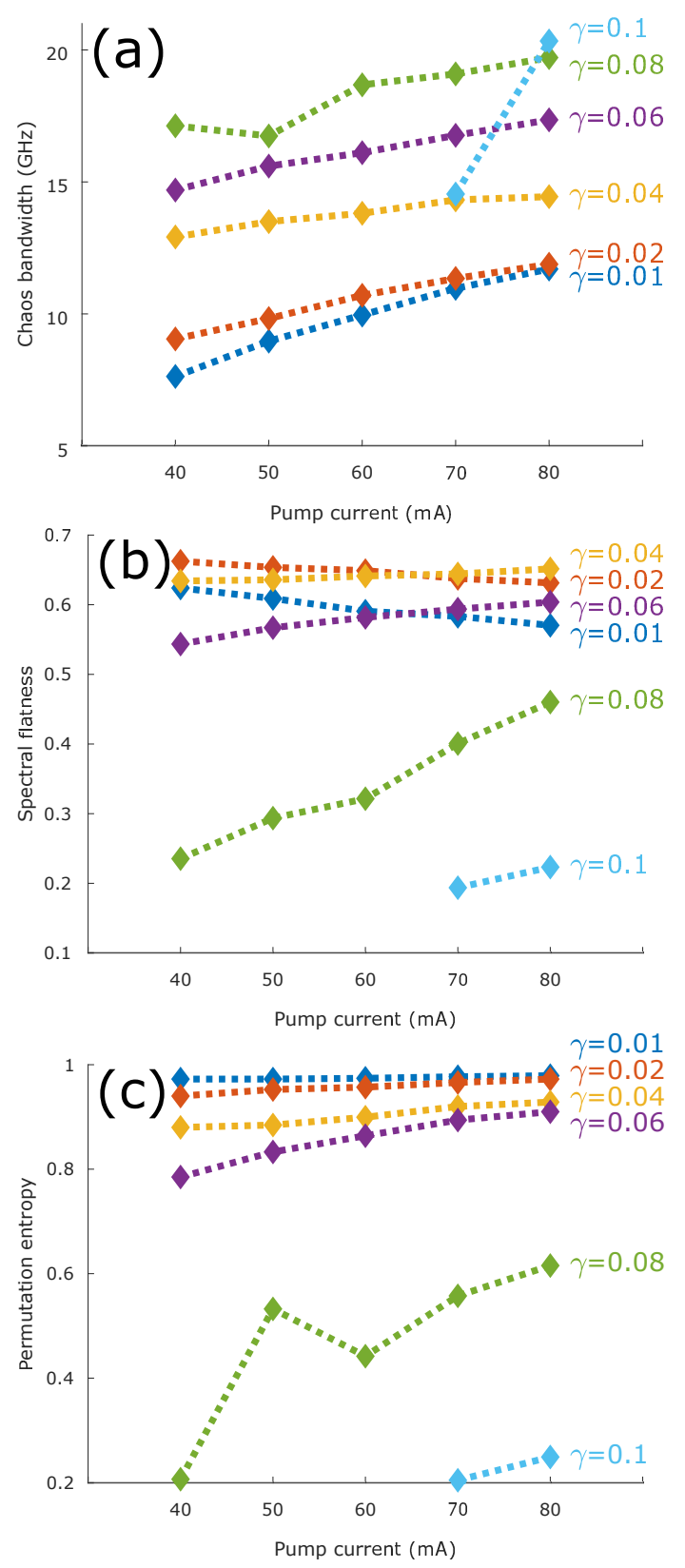

FIG. 8. Simulated evolution of (a) the chaos bandwidth, (b) the spectral flatness and (c) the $\operatorname{PE}(\tau)$, versus the pump current $(I)$, for five values of feedback strength $(\gamma)$. The delay is equal to $3.55 \mathrm{~ns}$.

flatness and $\operatorname{PE}(\tau)$ described in section IIIB will occur at a lower value of $\gamma$ as the pump current decreases. This explains why the $\operatorname{PE}(\tau)$ and the spectral flatness strongly increase with the pump current for $\gamma>0.06$.

\section{CONCLUSIONS}

Using an experiment with a self-pumped photorefractive phase-conjugate mirror and an accurate filtered model of PCF, we have been able to observe the influence 
of the external cavity length and of the pump current on the bandwidth and complexity of a laser with PCF. Large chaos bandwidth, spectral flatness and $\operatorname{PE}(\tau)$ are found experimentally, independently of the delay. On the other hand, the chaos bandwidth can be enhanced by an increase of the pump current while $\operatorname{PE}(\tau)$ and spectral flatness remain at high values. Our extensive parametric study of the chaos from PCF therefore show how PCF can be used to optimize the chaos properties of a laser system. Most of the experimental observations are well supported by numerical simulations.

There have been only few such in-depth analysis of chaos properties in a laser with feedback. The achieved performances are well beyond those found for a laser with $\mathrm{COF}$, hence confirming the superior performance of chaos generated by PCF. It is also worth noting that the bandwidth recorded here reaches about 3-4 times the relaxation oscillation frequency. Furthermore, for the majority of the parameter range, the values reach the limit of what can be captured by our state-of-the-art measurement setup. The chaos flatness measured is also close to nature of white noise but is obtained through a deterministic chaos mechanism. Additional improvement of bandwidth is expected with an additional optical injection, i.e., a well-known technique used for $\mathrm{COF}$ and therefore, an interesting path to consider next for PCF.

\section{ACKNOWLEDGMENTS}

Chaire photonique is funded by: Ministère de lEnseignement supérieur, de la Recherche et de IInnovation, Région Grand-Est, Département Moselle, European Regional Development Fund (ERDF), Metz Métropole, Airbus GDI Simulation; CentraleSupélec; Fondation CentraleSupélec
[1] G. D. VanWiggeren and R. Roy, Science 279, 1198 (1998).

[2] A. Uchida, K. Amano, M. Inoue, K. Hirano, S. Naito, H. Someya, I. Oowada, T. Kurashige, M. Shiki, S. Yoshimori, K. Yoshimura, and P. Davis, Nature Photonics 2, 728 (2008).

[3] F. Y. Lin and J. M. Liu, IEEE Journal on Selected Topics in Quantum Electronics 10, 991 (2004).

[4] M. Sciamanna and K. A. Shore, Nature Photonics 9, 151 (2015), arXiv:1507.08825.

[5] B. Krauskopf, G. R. Gray, and D. Lenstra, Physical Review E 58, 7190 (1998).

[6] J. S. Lawrence and D. M. Kane, Physical Review A 63, 1 (2001).

[7] É. Mercier, L. Weicker, D. Wolfersberger, D. M. Kane, and M. Sciamanna, Optics Letters 42, 306 (2017).

[8] T. Erneux, A. Gavrielides, K. Green, and B. Krauskopf, Physical Review E 68, 066205 (2003).

[9] É. Mercier, D. Wolfersberger, and M. Sciamanna, Scientific Reports 6, 18988 (2016).

[10] T. Malica, G. Bouchez, D. Wolfersberger, and M. Sciamanna, Optics Letters 45, 819 (2020).

[11] F. Y. Lin and J. M. Liu, Optics Communications 221, 173 (2003).

[12] R. Sakuraba, K. Iwakawa, K. Kanno, and A. Uchida, Optics Express 23, 1470 (2015).

[13] A. Argyris, D. Syvridis, L. Larger, V. Annovazzi-Lodi, P. Colet, I. Fischer, J. García-Ojalvo, C. R. Mirasso, L. Pesquera, and K. A. Shore, Nature 438, 343 (2005).

[14] K. Schires, S. Gomez, A. Gallet, G.-h. Duan, and F. Grillot, IEEE Journal of Selected Topics in Quantum Electronics 23, 1 (2017).

[15] L. Qiao, T. Lv, Y. Xu, M. Zhang, J. Zhang, T. Wang, R. Zhou, Q. Wang, and H. Xu, Optics Letters 44, 5394 (2019).

[16] A. Wang, Y. Yang, B. Wang, B. Zhang, L. Li, and Y. Wang, Optics Express 21, 8701 (2013).

[17] D. Rontani, E. Mercier, D. Wolfersberger, and M. Scia- manna, Optics Letters 41, 4637 (2016).

[18] K. Hirano, K. Amano, A. Uchida, S. Naito, M. Inoue, S. Yoshimori, K. Yoshimura, and P. Davis, IEEE Journal of Quantum Electronics 45, 1367 (2009).

[19] G. Bouchez, C.-H. Uy, B. Macias, D. Wolfersberger, and M. Sciamanna, Optics Letters 44, 975 (2019).

[20] K. Petermann, IEEE Journal on Selected Topics in Quantum Electronics 1, 480 (1995).

[21] J. Feinberg, Optics Letters 7, 486 (1982).

[22] O. K. Andersen, A. P. Fischer, I. C. Lane, E. Louvergneaux, S. Stolte, and D. Lenstra, IEEE Journal of Quantum Electronics 35, 577 (1999).

[23] M. Virte, A. Karsaklian Dal Bosco, D. Wolfersberger, and M. Sciamanna, Physical Review A 84, 1 (2011).

[24] A. Gray and J. Markel, IEEE Transactions on Acoustics, Speech, and Signal Processing 22, 207 (1974).

[25] C. Bandt and B. Pompe, Physical Review Letters 88, 174102 (2002).

[26] M. Riedl, A. Müller, and N. Wessel, The European Physical Journal Special Topics 222, 249 (2013).

[27] É. Mercier, C.-H. Uy, L. Weicker, M. Virte, D. Wolfersberger, and M. Sciamanna, Physical Review A 94, 061803 (2016).

[28] L. Weicker, C.-H. Uy, D. Wolfersberger, and M. Sciamanna, Chaos: An Interdisciplinary Journal of Nonlinear Science 27, 114314 (2017).

[29] T. Rauch, C. Denz, and T. Tschudi, Optics Communications 88, 160 (1992).

[30] K. Green and B. Krauskopf, Physical Review E 66, 016220 (2002).

[31] X. Guo, T. Liu, L. Wang, X. Fang, T. Zhao, M. Virte, and Y. Guo, Optics Express 28, 1238 (2020), 1909.10160.

[32] L. Weicker, T. Erneux, D. Wolfersberger, and M. Sciamanna, Physical Review E 92, 022906 (2015).

[33] G. P. Agrawal and J. T. Klaus, Optics Letters 16, 1325 (1991).

[34] D. DeTienne, G. Gray, G. Agrawal, and D. Lenstra, IEEE Journal of Quantum Electronics 33, 838 (1997). 